\title{
Exploring risk factors of hepatitis B virus-associated hepatocellular carcinoma: prospective verse retrospective studies
}

\author{
Guangwen Cao
}

Received: 4 August 2010/ Accepted: 9 August 2010/Published online: 7 September 2010

(C) Springer 2010

Major risk factors for hepatocellular carcinoma (HCC) vary by region and population. Chronic infection with hepatitis $\mathrm{B}$ virus (HBV) and/or hepatitis $\mathrm{C}$ virus (HCV), heavy alcohol intake, aflatoxin exposure, haemochromatosis, nonalcoholic fatty liver disease, obesity, diabetes mellitus and tobacco smoking have been suggested to be risk factors of HCC. However, chronic infection with HBV remains the major cause of $\mathrm{HCC}$ in the world. More than $50 \%$ of HCC cases worldwide and $80 \%$ of HCC cases in most high-risk areas, such as the Asia-Pacific region and Africa, are attributable to HBV infection. The relative risk of HCC among individuals infected with HBV ranges from 5 to 49 in case-control studies and from 7 to 98 in cohort studies [1].

In HBV endemic regions, $\mathrm{HBV}$ infection is usually acquired perinatally or in childhood, whereas in HBV nonendemic areas, it is mostly transmitted during adolescence or adulthood. Newborns become chronic HBV carriers at a very high rate (about 90\%), while immune-competent adults are generally described as developing chronic infection at a rate of $5-10 \%$. HBV subgenotype $\mathrm{C} 2$ is more prone to causing chronic infection than HBV subgenotype B2 following acute hepatitis B [2]. Up to $40 \%$ of patients with chronic HBV infection will develop the life-threatening complications of HCC or decompensated liver cirrhosis (LC). Chronic hepatitis B (CHB), LC and HCC are progressive liver diseases and consecutive stages of HBV-

The online version of the article referred to in this editorial can be found under doi:10.1007/s00535-010-0307-4.

G. Cao $(\bowtie)$

Department of Epidemiology, Second Military Medical University, 800 Xiangyin Rd., Shanghai 200433, China

e-mail: gcao@smmu.edu.cn associated hepatocarcinogenesis, although CHB may precede the asymptomatic hepatitis $\mathrm{B}$ surface antigen (HBsAg) carrier (ASC) state and HCC does not have to pass through the CHB or LC stage. Hepatitis B e antigen (HBeAg) expression indicates active viral replication. $\mathrm{HBeAg}$ expression, high viral load and alanine aminotransferase (ALT) levels are associated with an increased risk of HCC. HBV subgenotypes $\mathrm{B} 2$ and $\mathrm{C} 2$ are endemic in most parts of Asia, while subgenotype $\mathrm{B} 1$ is endemic in Japan. Chronic infection with HBV C2 is frequently associated with an increased risk of LC and HCC in patients older than 50 years, whereas chronic infection with HBV B2 is associated with HCC or HCC recurrence in young, mostly non-cirrhotic, patients. In comparison with infection with HBV B2 or C2, infection with HBV B1 is associated with fulminant hepatitis $\mathrm{B}$, a lower incidence of HCC and the development of HCC when older. Different HBV genotypes display their own distinct mutation pattern in the preS region and in the enhancer II (EnhII)/basic core promoter $(\mathrm{BCP}) /$ precore region of the $\mathrm{HBV}$ genome. Takahashi and colleagues identified HCC-associated HBV mutations through the comparative analysis of full-length HBV isolates (95\% genotype C) from sera of 40 Japanese patients with HCC. They found that deletions and missense mutations in the preS2 region, A1762T and G1764A (often appearing simultaneously, termed A1762T/G1764A) and T1753C/A mutations in the BCP region and G1613A and C1653T mutations in the EnhII region were more frequent in HCC patients [3]. This pioneering work has aroused great interest in the field. Many studies pertaining to the association of HBV mutations with the risk of HCC have been published during the past decade. It has been found that the HBV preS deletion, A1762T/G1764A, T1753V, $\mathrm{C} 1653 \mathrm{~T}$ and $\mathrm{T} 31 \mathrm{C}$ are each associated with a significantly increased risk of $\mathrm{HCC}$ [4]. Of the two major $\mathrm{HBV}$ 
subgenotypes endemic in Asia, HBV $\mathrm{C} 2$ has higher frequencies of $\mathrm{HCC}$-associated HBV mutations, including the preS deletion, A1762T/G1764A, T1753V and T31C, than HBV B2. However, these studies vary in the quality of their design and conduct. Most of the designs are clinicbased case-control or cross-sectional studies; few are population-based, prospective studies.

Retrospective studies are only able to present a statistical relationship between exposures and the risk of $\mathrm{HBV}$-associated HCC, while population-based prospective studies with comparison group(s) are more reliable than retrospective studies in elucidating a causal relationship between exposures and hepatocarcinogenesis. There are two kinds of prospective studies: the cohort study and the nested case-control study. The cohort study is a type of study in which the incidence of HCC during the follow-up is compared between $\mathrm{HBV}$-infected participants with or without HBV variables and other exposures (exposure) in a given cohort, with exposure being defined in terms of the data collected at baseline prior to HCC development. The nested case-control study refers to a study in which the HBV-infected participants who develop HCC (cases) during the follow-up are selected and compared with those without HCC (controls) from the same cohort of HBV carriers, with the data on exposure being documented at baseline, just as in the cohort study. Theoretically, the results from the cohort study should be more reliable than those from the nested case-control study in terms of elucidating the causal relationship.

The population-based cohort study by Kusakabe et al. [5] in this issue of the Journal of Gastroenterology has, for the first time, demonstrated that the A1762T/G1764A mutation (multivariable-adjusted hazard ratio 7.05, 95\% confidence interval 1.03-48.12) is a uniquely independent risk factor of $\mathrm{HCC}$ in $\mathrm{HBV}$ mono-infected subjects in Japan. The several advantages of this study should be addressed. First, A1762T/G1764A, mostly found in HBV $\mathrm{C} 2$, is shown to be an independent risk factor associated with the risk of HCC during the natural course of HBV infection in an area with a low prevalence of HBV C2. This result was confirmed by the survival analysis. The A1762T/ G1764A mutation is therefore a reliable HBV variable for classifying high-risk $\mathrm{HBV}$-infected subjects prior to the development of HCC. Second, HBV B1 was found to have lower frequencies of $\mathrm{HCC}$-associated $\mathrm{HBV}$ mutations, including A1762T/G1764A, T1753V and C1653T, than HBV C2, implying that HBV B1 is less associated with the risk of $\mathrm{HCC}$ than $\mathrm{HBV} \mathrm{C} 2$, although the difference does not reach the significance level. Third, the risk factors identified in the univariate analysis are indicative of the prevention and control of $\mathrm{HCC}$ in the HBV mono-infected subjects. Data from clinical-based cohort studies are used to establish clinical scoring systems to predict HCC in chronic hepatitis B carriers. The independent risk factors of $\mathrm{HCC}$, such as male gender, age ( $>40$ years), high HBV DNA level, low albumin level, low platelets levels, core promoter mutations and cirrhosis, have been selected as components of the scoring systems [6]. Data from this study and other population-based prospective studies may be summarized to establish a community-based scoring system to predict HCC in HBV-infected population.

One of my concerns regarding this study is the design of the epidemiological study for the elucidation of the risk factor of HCC. Chronic infection with HCV, rather than with HBV, is the leading etiological agent of HCC in Japan [7]. The prevalence of HBV mono-infection in the study population aged 40-69 years at the time of the baseline survey was only $2.5 \%$. The design, scale and even the cost of this study might be similar to those of the cohort study carried out in Taiwan where the prevalence of HBsAg is reported to be between 10 and 20\% [8]. Only 13 HCC cases among $479 \mathrm{HBV}$ mono-infected subjects were found in this study. This embarrassing number of HCC cases among the HBV mono-infected subjects leads to weak statistical power. In addition, 56\% (268/479) of the mono-HBV infected subjects are infected with HBV B1, while HBV B1 may be less associated with the risk of HCC than HBV C2. Thus, this cohort study carried out in a population with a low rate of exposure (chronic infection with HBV, especially with HBV C2) should not be cost-effective. An ageand sex-matched/-adjusted case-control study would be more preferable than a cohort study for elucidating the association of HBV variables with the risk of HCC in areas with a low prevalence of HBV infection. Another concern pertaining to cohort studies published to date, including this one, is that exposure data are documented based on only one time-point at entry. During chronic HBV infection, viral load and $\mathrm{HBeAg}$ expression tend to fluctuate with increasing age [9]. The HCC-associated HBV mutants, such as the A1762T/G1764A mutants, could not be transmitted via mother-to-child transmission because the children whose mothers carrying HBV mutants were mostly found to be infected with the wild-type form of the same viruses [10]. In adolescents or adults, HBV tends to transmit in the $\mathrm{HBeAg}$-positive period when the serum viral load is high [2]. HBeAg seroconversion has been associated with the presence of mutations in the BCP and/ or precore regions. The HCC-associated mutations are likely selected by HBV-host interaction during long-term HBV-induced carcinogenesis. The HCC-associated mutations in $\mathrm{BCP}$ and preS deletions then gradually accumulate during hepatocarcinogenesis and eventually become the predominant or exclusive viral population at the stage of HCC. Our recent study revealed that preS deletions, C1653T, T1753V and A1762T/G1764A are increasingly more prevalent as the chronic $\mathrm{HBV}$ infection progresses 
from the ASC state to LC or HCC and that the mutations in combination are specific for HCC [4]. Published cohort studies have confirmed that A1762T/G1764A alone, a possible early mutation event in hepatocarcinogenesis, is independently associated with an increased risk of HCC, possibly because exposure data are only documented based on one time-point at entry. Sequential examination of the mutations in $\mathrm{CP}$ and/or preS regions of $\mathrm{HBV}$ may provide more accurate information than one time-point survey at entry for the prediction of the development of HCC. The retrospective study, which is preferably used in populations with a low rate of exposure, usually provide a wide range of HBV variables and other risk factors statistically associated with the risk of HCC, whereas the prospective study, which is frequently applied in populations with a high rate of exposure, is able to confirm the casual relationship between the risk factors from the case-control study and $\mathrm{HCC}$ and discriminate the $\mathrm{HBV}$-infected subjects who more likely develop HCC in the future. Thus, the prospective study and the retrospective one have complementary advantages and should be reasonably integrated in the exploration of the casual relationship between risk factors and the risk of $\mathrm{HBV}$-associated HCC.

\section{References}

1. Nguyen VT, Law MG, Dore GJ. Hepatitis B-related hepatocellular carcinoma: epidemiological characteristics and disease burden. J Viral Hepat. 2009;16:453-63.
2. Zhang HW, Yin JH, Li YT, Li CZ, Ren H, Gu CY, et al. Risk factors for acute hepatitis B and its progression to chronic hepatitis in Shanghai, China. Gut. 2008;57:1713-20.

3. Takahashi K, Akahane Y, Hino K, Ohta Y, Mishiro S. Hepatitis B virus genomic sequence in the circulation of hepatocellular carcinoma patients: comparative analysis of 40 full-length isolates. Arch Virol. 1998;143:2313-26.

4. Liu S, Zhang H, Gu C, Yin J, He Y, Xie J, et al. Associations between hepatitis $\mathrm{B}$ virus mutations and the risk of hepatocellular carcinoma: a meta-analysis. J Natl Cancer Inst. 2009;101: 1066-82.

5. Kusakabe A, Tanaka Y, Inoue M, Kurbanov F, Tatematsu K, Nojiri S, et al. A population-based cohort study for the risk factors of HCC among hepatitis B virus mono-infected subjects in Japan. J Gastroenterol. 2010. doi:10.1007/s00535-010-0307-4.

6. Yuen MF, Tanaka Y, Fong DY, Fung J, Wong DK, Yuen JC, et al. Independent risk factors and predictive score for the development of hepatocellular carcinoma in chronic hepatitis B. J Hepatol. 2009;50:80-8.

7. Umemura T, Ichijo T, Yoshizawa K, Tanaka E, Kiyosawa K. Epidemiology of hepatocellular carcinoma in Japan. J Gastroenterol. 2009;44[Suppl 19]:102-7.

8. Yang HI, Yeh SH, Chen PJ, Iloeje UH, Jen CL, Su J, et al. Associations between hepatitis B virus genotype and mutants and the risk of hepatocellular carcinoma. J Natl Cancer Inst. 2008;100:1134-43.

9. Yin J, Zhang H, He Y, Xie J, Liu S, Chang W, et al. Distribution and hepatocellular carcinoma-related viral properties of hepatitis B virus genotypes in Mainland China: a community-based study. Cancer Epidemiol Biomarkers Prev. 2010;19:777-86.

10. Cheng H, Su H, Wang S, Shao Z, Men K, Li M, et al. Association between genomic heterogeneity of hepatitis B virus and intrauterine infection. Virology. 2009;387:168-75. 